\title{
CORRESPONDENCE
}

\section{The Diagnosis and Treatment of Acute Pulmonary Embolism}

by Dr. med. Alexander Schellhaaß, Prof. Dr. med. Andreas Walther, Prof. Dr. med. Stavros Konstantinides, Prof. Dr. med. Bernd W. Böttiger in volume $34-35 / 2010$

\section{Don't Forget Scintigraphy in Pulmonary Embolism}

In their review article (1) the authors recommended a diagnostic algorithm for the scenario of suspected embolism of the pulmonary artery. Lung ventilation and perfusion scintigraphy is mentioned only as an aside, and the authors say that multidetector computed tomography has taken over as the gold standard imaging method. They back up their statement with a 2008 guideline from the European Society of Cardiology (2). However, in the cited guideline, the value of scintigraphy is explained in a rather more differentiated manner. The guideline emphasizes in particular the high negative predictive value of a normal perfusion scintigram. A prospective multicenter study, which is often cited as a justification for the use of spiral computed tomography (CT), has also again confirmed the safety of diagnostic algorithms while using scintigraphy (3).

We would also ask that more attention be paid to the possible contraindications and side effects of the contrast media required for $\mathrm{CT}$ scanning (renal failure, hyperthyroidism, contrast medium allergy). These may even be life threatening. Scintigraphy is a valuable alternative, but the way the information is presented runs the risk that it is all but forgotten about.

Mentioning technical progress in nuclear medical imaging with three-dimensional assessment of perfusion and ventilation - also when using the SPECT/CT technique - may exceed the remit of the review article. However, all these contribute to making the procedure safe.

The effective dose usually does not matter in an emergency situation. CT and scintigraphy do not differ much in that setting. But the dose to parts of the body should be considered, as well as the dose to the breast in young women and that to the uterus in pregnant women in their third trimester.

Even though the review article aimed to provide a simple algorithm of diagnostic procedures, we hope to have made a valuable contribution to the material. DOl: 10.3238/arztebl.2011.0142a

\section{REFERENCES}

1. Schellhaaß A, Walther A, Konstantinides St, Böttiger BW: The diagnosis and treatment of acute pulmonary embolism. Dtsch Arztebl Int 2010; 107(34-35): 589-95.

2. Torbicki A, Perrier A, Konstantinides S: Task Force for the Diagnosis and Management of Acute Pulmonary Embolism of the European

Society of Cardiology. Guidelines on the diagnosis and management of acute pulmonary embolism: the Task Force for the Diagnosis and Management of Acute Pulmonary Embolism of the European Society of Cardiology (ESC). Eur Heart J 2008; 29: 2276-315.

3. Anderson DR, Kahn SR, Rodger MA, et al.: Computed tomographic pulmonary angiography vs ventilation-perfusion lung scanning in patients with suspected pulmonary embolism: a randomized controlled trial. JAMA 2007; 298: 2743-53.

Univ.-Prof. Dr. med. Dr. rer. nat. Otmar Schober Univ.-Prof. Dr. med. Hermann-Josef Pavenstädt

Prof. Dr. med. Matthias Weckesser

Klinik und Poliklinik für Nuklearmedizin

Universitätsklinikum Münster

Albert-Schweitzer-Str. 33, 48149 Münster, Germany

weckesm@uni-muenster.de

\section{Don't Forget Ventilation-Perfusion Scintigraphy}

The authors of the article seem to be under the impression that multidetector computed tomography (MDCT) has completely taken over from ventilation-perfusion scintigraphy in diagnosing acute pulmonary embolism.

It is certainly correct that MDCT is more readily available in emergency situations (especially during night shifts), but limitations often arise owing to the necessary administration of iodine containing contrast medium. In patients with allergies to contrast medium, different thyroid dysfunctions, and renal failure, administration of contrast medium, and therefore MDCT, is often not possible. Especially in these cases, ventilation-perfusion scintigraphy is the method of choice for diagnosing acute pulmonary embolism, as it is non-invasive, low-risk, and tried and tested. An embolism can be detected with a sensitivity of $96-97 \%$ and a specificity of $90-95 \%$.

DOI: 10.3238/arztebl.2011.0142b

\section{REFERENCES}

1. Pöppel TD, Krause BJ: Pulmonale Perfusions-Ventilationsszintigrafie in der Diagnostik der Lungenembolie. Radiologe 2007; 47: 698-707.

2. Schellhaaß A, Walther A, Konstantinides St, Böttiger BW: The diagnosis and treatment of acute pulmonary embolism. Dtsch Arztebl Int 2010; 107(34-35): 589-95.

\section{Dr. med. Holger Trötsche}

Danziger Str. 29 B, 49324 Melle, Germany

Holger.Troetschel@t-online.de

\section{Remember Transthoracic Ultrasonography}

The article has great relevance to clinical practice and reflects the prevailing opinion in Germany (1). However, in their literature review, the authors unfortunately did not come across the very readable meta-analysis by T Niemann from University Hospital Basel, on the importance of transthoracic ultrasonography (TUS) in the diagnostic evaluation of acute pulmonary embolism (2). This meta-analysis shows a sensitivity of $80 \%$ and 
a specificity of $93 \%$ if only prospective studies are included; these results are no worse than those obtained by multidetector computed tomography (MDCT) in diagnosing pulmonary embolism.

Minimizing exposure to radiation has become an important topic in radiology, especially since CT scanning was introduced, and European initiatives are searching for alternative diagnostic methods (SPECT-protocol). TUS is the obvious choice in this setting, since it is available everywhere and easy to learn, as well as offering valid results. In emergency units and intensive care wards in patients who cannot be transported it even has advantages compared with MDCT. A further advantage lies in the fact that it is possible using one soundhead to expose the deep leg veins (source), the right heart (place of transmission), and the lung as the target organ of the embolism. If one assumes a combination of deep vein thrombosis in the leg with acute pulmonary embolism in $80 \%$ of cases, this is enormously important for rapid initiation of anticoagulation. Pneumologists have learnt TUS in recent years, and the procedure has become a standard tool in pneumology clinics.

Unfortunately, TUS has hitherto not been included in any national or international guidelines. This really should change sooner rather than later.

DOI: 10.3238/arztebl.2011.0142c

\section{REFERENCES}

1. Schellhaaß A, Walther A, Konstantinides St, Böttiger BW: The diagnosis and treatment of acute pulmonary embolism. Dtsch Arztebl Int 2010; 107(34-35): 589-95.

2. Niemann T, Engelhof T, Bongartz G: Transthoracic sonography for the detection of pulmonary embolism - a Meta-Analysis. Ultraschall in Med 2009; 30: 150-6.

3. Brenner DJ, Hall EJ: Computed tomography — an increased source of radiation. N Engl J Med 2007; 357: 2277-84.

\section{Prof. Dr. med. Detlef Kirsten}

Krankenhaus Großhansdorf

Zentrum für Pneumologie und Thoraxchirurgie

Wöhrendamm 80, 22927 Großhansdorf, Germany

DetlefKirsten@online.de

\section{Regulation in Germany Is Lacking}

For simplicity's sake, the only imaging technique mentioned in the context of diagnosing pulmonary embolism in this article is multidetector computed tomography (MDCT). This ignores the uncertain data situation regarding its sensitivity and possible risks for the patients. Only one-third of non-radiologists in Germany are informed about the enormously high radiation exposure associated with MDCT (7-30 mSv depending on device and protocol, even higher radiation exposure of the breasts), whereas in the United States the use of MDCT is already subject to rules and regulations. Further developments (iterative reconstruction) can only alleviate the problem, not eliminate it. Collateral damage due to MDCT (29 000 cases of cancer predicted, 14500 deaths per year in the US, 1.5-2\% additional cases of cancer in the long term) would be tolerable only if there were no alternatives to MDCT.
European guidelines for the diagnostic evaluation of pulmonary embolism recommend the tomographic variant of scintigraphy (ventilation[V]/Perfusion[P]-SPECT), which has a higher sensitivity than MDCT (1). There is no problem with specificity, as postulated in the US 20 years ago, which is confirmed by comparison with MDCT (2). As a result, every positive finding (from two subsegments) is clinically evaluable. Many patients with suspected non-high-risk pulmonary embolism have mild to moderate symptoms and diagnostic evaluation usually takes place only days after the onset of their problems. There is enough time to exclude pulmonary embolism by using V/Q-SPECT. In patients with more severe symptoms, MDCT is indicated in the emergency setting for the purposes of differential diagnostic evaluation. In future, V/P-SPECT/low-dose CT will be able to be used for this. Initial results in acute pulmonary embolism - sensitivity $97 \%$, specificity $100 \%$, sensitivity MDCT $68 \%$ (3) give cause for optimism.

DOl: 10.3238/arztebl.2011.0143a

\section{REFERENCES}

1. Bajc M, Neilly JB, Miniati M, et al.: EANM guidelines for ventilation/ perfusion scintigraphy: Part 1. Pulmonary imaging with ventilation/ perfusion single photon emission tomography. Eur J Nucl med Mol Imaging 2009; 36(8): 1356-70, Part 2. Algorithms and clinical considerations for diagnosis of pulmonary emboli with $\mathrm{V} / \mathrm{P}(\mathrm{SPECT})$ and MDCT. Eur J Nucl med Mol Imaging 2009; 36(9): 1528-38.

2. Schuemichen C: Pulmonary embolism: is multislice CT the method of choice? Against. Eur J Nucl Med Mol Imaging 2005; 32: 103-7.

3. Gutte H, Mortensen J, Jensen CV, et al.: Detection of pulmonary embolism with combined ventilation-perfusion SPECT and low-dose CT: head-to head comparison with multidetector CT angiography. J Nucl Med 2009; 50(12): 1987-92

4. Schellhaaß A, Walther A, Konstantinides St, Böttiger BW: The diagnosis and treatment of acute pulmonary embolism. Dtsch Arztebl Int 2010; 107(34-35): 589-95.

Prof. Dr. med. Carl Schümichen

Klinik und Poliklinik für Nuklearmedizin

Universitätsklinik Rostock

Gertrudenplatz 1

18057 Rostock, Germany

carl.schuemichen@med.uni-rostock.de

\section{Addenda Required}

The radiation dose of an investigation using multidetector spiral computed tomography (MDCT) with contrast medium is $5-8 \mathrm{mS}$, which equates to a risk of radiation cancer of $1: 10^{3}-1: 10^{4}(1)$. The article does not mention that when using MDCT, a pulmonary embolism is actually found in only $20-32 \%$ of patients in whom this is suspected.

$80-92 \%$ of patients with acute pulmonary embolism have a deep vein thrombosis of the leg. If a deep vein thrombosis is confirmed and pulmonary embolism is clinically suspected at the same time, then the German guidelines regard this as confirmed pulmonary embolism. Complete venous compression ultrasonography (CCUS) can be used to reliably identify deep vein thromboses in the calf and thigh, and we conduct CCUS as our primary diagnostic evaluation in 
suspected pulmonary embolism after scoring and, if required, D-dimer testing (2).

Transesophageal echocardiography, as mentioned in the article, successfully confirms central pulmonary embolism in less than $10 \%$ of patients in whom this diagnosis is suspected. A new method-endobronchial ultrasonography (EBUS) - is available, which was developed primarily for staging the mediastinal lymph nodes in bronchus carcinoma. Since the pulmonary artery branch, the right and left pulmonary arteries, and the lobar pulmonary artery extend alongside the bronchi at a distance of $1-2 \mathrm{~mm}$, it is possible to use this technique to confirm central pulmonary embolism - from the pulmonary valve to the lobar arteriesin more than $95 \%$ of patients (3). Only $15 \%$ of all symptomatic pulmonary embolisms are peripheral in isolation.

Endobronchial ultrasonography can be used in all patients with pulmonary embolism, whether high risk or low risk, and constitutes an alternative in renal failure, pregnancy, contrast medium allergy, hemodynamic instability in intensive care, and in patients refusing angio-CT because of the radiation exposure. The method is yet to be evaluated in multicenter studies. DOl: 10.3238/arztebl.2011.0143b

\section{REFERENCES}

1. David J, Brenner E, Hall J: Computed tomography — an increasing source of radiation exposure. N Engl J Med 2007; 357: 2277-84.

2. Schellong SM: Venous ultrasonography in symptomatic and asymptomatic patients: an updated review. Current Opinion in Pulmonary Medicine 2008; 14: 374-80.

3. Aumiller J, Herth FJ, Krasnik M, Eberhardt R: Endobronchial ultrasound for detecting central pulmonary emboli: a pilot study. Respiration 2009; 77: 298-302.

4. Schellhaaß A, Walther A, Konstantinides St, Böttiger BW: The diagnosis and treatment of acute pulmonary embolism. Dtsch Arztebl Int 2010; 107(34-35): 589-95.

\section{Dr. med. Josef Aumiller}

Marienkrankenhaus

Zentrum Innere Medizin

Schwerpunkt: Kardiologie, Pneumologie,

Angiologie, Endokrinologie

Alfredstr. 9, 22087 Hamburg, Germany

aumiller.innere@marienkrankenhaus.org

\section{In Reply:}

As we mentioned in our article, many methods, or combinations therefore, are available for the reliable diagnostic confirmation or exclusion of pulmonary embolism (1). A more detailed description of the individual investigative methods would have exceeded the remit of a review article.

Weckesser, Trötschel, and Schümichen emphasize the importance of perfusion and ventilation scinti- graphy. We agree that scintigraphy is a useful alternative to MDCT if relative contra-indications to the administration of contrast medium exist, as long as the equipment is sufficiently close to be immediately available, even in emergency situations. However, a recent survey showed that ventilation-perfusion scintigraphy is not available round the clock in $23 \%$ of participating radiological departments, whereas MDCT is not available $24 / 7$ in only $3 \%$ of participating departments (2). It also deserves mention that non-iodine containing contrast media can be administered in most cases of contrast medium allergy, thyroid dysfunctions, or renal failure. In this context we wish the point out the standard operating procedures (SOP) for contrast medium administration at Heidelberg University Hospital (3).

The results of the meta-analysis mentioned by Kirsten about the importance of transthoracic ultrasonography (TUS) in the diagnostic evaluation of acute pulmonary embolism were not reproduced in a recent study (4). Because of the unclear data situation it therefore currently seems unjustified to recommend its use outside study settings. The same is true for endobronchial ultrasonography (EBUS).

We thank Aumiller for his comment about sonography of the leg veins. In hemodynamically stable patients with suspected pulmonary embolism, this diagnosis is regarded as confirmed if sonography shows a venous thrombosis in the leg (5).

DOl: 10.3238/arztebl.2011.0144

\section{REFERENCES}

1. Schellhaaß A, Walther A, Konstantinides St, Böttiger BW: The diagnosis and treatment of acute pulmonary embolism. Dtsch Arztebl Int 2010; 107(34-35): 589-95.

2. Bhargavan M, Sunshine JH, Hervey SL, Jha S, Vializ J, Owen JB: The actual role of CT and ventilation-perfusion scanning in workup for suspected pulmonary embolism: evidence from hospitals. AJR Am J Roentgenol 2009; 193: 1324-32.

3. www.klinikum.uni-heidelberg.de/fileadmin/radiologie/radiodiagnos tik/SOP_S/SOP_KM_Gabe_18_10_2010.pdf

4. Pfeil A, Reissig A, Heyne JP, Wolf G, Kaiser WA, Kroegel C, Hansch A Transthoracic sonography in comparison to multislice computed tomography in detection of peripheral pulmonary embolism. Lung 2010; 188: 43-50

5. Interdisziplinäre S2-Leitlinie: Diagnostik und Therapie der Venenthrombose und der Lungenembolie. Vasa 2010; 39: S78.

\section{Dr. med. Alexander Schellhaaß}

Klinik für Anaesthesiologie

Universitätsklinikum Heidelberg

Im Neuenheimer Feld 110, 69120 Heidelberg, Germany

Alexander.Schellhaass@med.uni-heidelberg.de

\section{Conflict of interest statement}

The authors of all contributions declare that no conflict of interest exists according to the guidelines of the International Committee of Medical Journal Editors. 